\title{
Diagnóstico de responsabilidad social universitaria en la Escuela Profesional de Administración de una universidad privada de Arequipa
}

\section{Diagnosis of university social responsibility in the Professional School of Administration of a private university of Arequipa}

\author{
Sandra Gómez Rodríguez \\ Universidad Católica San Pablo, Arequipa, Perú \\ sgomez@ucsp.edu.pe \\ Paola Pinto de Alarcón \\ Universidad Católica San Pablo, Arequipa, Perú \\ papinto@ucsp.edu.pe
}

\section{Resumen}

El objetivo de esta investigación es medir el nivel de cumplimiento de la responsabilidad social universitaria (RSU) en la Escuela de Administración de una universidad privada de Arequipa (UPA), con los instrumentos elaborados por Baca, Rondán y García del Junco (2017) y su método sistémico. Se concluyó que las autoridades de la Escuela de Administración de la UPA perciben que el $42.86 \%$ de los estándares no son cumplidos, el $41.76 \%$ de los estándares son considerados como oportunidades de mejora y el 15.38\% de los estándares se cumplen plenamente. Por otro lado, la interacción con actividades de RSU de los stakeholders internos de la UPA es el siguiente: el 55.63\% interactúa mucho y siempre, el $26.85 \%$ interactúa poco y el $17.52 \%$ no interactúa nunca. Las dimensiones que registran diferencias significativas en sus medias entre las distintas categorías de stakeholders son gestión organizacional, gestión ambiental y docencia.

\section{Palabras clave}

Responsabilidad social universitaria - stakeholders internos - percepción 


\begin{abstract}
The purpose of this study is to measure the level of compliance regarding university social responsibility (USR) in the School of Administration of a private university of Arequipa (PUA), with the instruments developed by Baca, Rondán and García del Junco (2017) and their systemic method. It was concluded that the authorities of the School of Administration of the PUA perceive that $42.86 \%$ of the standards are not met, $41.76 \%$ of the standards are considered opportunities for improvement and $15.38 \%$ of the standards are fully met. The interaction of internal stakeholders with USR activities is as follows: $55.63 \%$ interact often or always, $26.85 \%$ have little interaction, and $17.52 \%$ have no interaction. USR dimensions that obtained significant differences in their means when comparing different stakeholder categories were organizational management, environmental management and teaching.
\end{abstract}

\title{
Keywords
}

University social responsibility - internal stakeholders - perception

\section{Introducción}

La responsabilidad social universitaria (RSU) es un tema que ha cobrado vital importancia en el desarrollo de instituciones educativas de nivel superior en el mundo y en la última década en el Perú.

La Organización de las Naciones Unidas para la Educación, la Ciencia y la Cultura (Unesco) definió a la universidad como aquel espacio de formación de personas y futuros profesionales que busca desarrollar, en ellos, el mejor desempeño laboral que les permita ejercer una ciudadanía responsable (Unesco, 2009). Por ello, es importante la formación en conocimientos y sobre todo en principios éticos que respalden la formación académica impartida. En los años noventa se creó el Instituto Internacional para la Educación Superior en América Latina y el Caribe (IESALC), institución encargada de desarrollar la Cátedra Unesco, así como temas de Responsabilidad Social Universitaria y la implicancia de este a nivel de América Latina y el Caribe. En este contexto, se crea el Observatorio Regional de Responsabilidad Social para América Latina y el Caribe (ORSALC), mencionado en el portal del IESALC, en el que se detallan las diversas actividades que se han desarrollado como parte del trabajo en la difusión de la responsabilidad social universitaria en la región.

Por otro lado, la encíclica Ex Corde Ecclesiae menciona la misión de la Universidad como la «constante búsqueda de la verdad mediante la investigación, la conservación y la comunicación del saber para el bien de la sociedad», dirigiendo las labores de una universidad respecto a su actuar y misión dentro de la sociedad en la que se desenvuelve (Juan Pablo II, 1990). 
Para Gil (2013) la RSU recae en las instituciones de nivel superior. Debido al cambio social y ambiental por el que atraviesa el mundo, la RSU exige plataformas educativas que tengan un rol protagónico en la generación de propuestas de solución para este tipo de problemas. Es por ello que, en el ámbito mundial, las universidades no deben solo limitarse al contexto de la enseñanza, sino también a fomentar acciones de responsabilidad social, para la formación de sus alumnos tanto académica como éticamente. Asimismo, De la Calle (2010) concluye que los alumnos deben sensibilizarse, desde las aulas universitarias, en temas de responsabilidad social para que, de esta manera, sean conscientes de la responsabilidad que asumen como miembros de la sociedad.

La responsabilidad social, en una primera instancia, fue estudiada dentro de las organizaciones, cooperaciones y empresas para luego pasar a un nivel educativo, según indican Mendoza y Salas (2015). En este punto, es importante tener claro cuáles son las principales funciones del trabajo que se realiza dentro de las universidades. Según Castañeda, Ruiz, Viloria, Castañeda y Quevedo (2007), podemos definir las siguientes: gestión, docencia, extensión e investigación. Por otro lado, Vallaeys (2008) también hace mención de la capacidad que deben desarrollar las universidades para proponer un conjunto de valores y creencias a través del desarrollo de cuatro procesos educativos clave: la investigación, extensión, docencia y gestión. Para Gaete (s.f.) las instituciones de «educación superior», en especial las universidades, realizan sus actividades en un «proceso de cambio social», que en algunas ocasiones no están alineadas con la misión de la universidad. El autor señala que la definición de la RSU está vinculada con valores y buenas prácticas que «expresan un compromiso real y específico con las necesidades y demandas de los grupos de interés».

La RSU también ha sido conceptualizada como proyección social o servicio social, por lo tanto, debe responder a la enseñanza-aprendizaje a la que está llamada la universidad, contribuyendo significativamente al bien común de la comunidad (Domínguez Granda y Rama, 2012).

Sin embargo, Vallaeys (2008) refiere que la RSU y todas las acciones que la engloban no «necesariamente» deben entenderse como sinónimos de «ayuda social» o «acción social». Más bien, según este autor, la RSU apunta a labores que fomenten un verdadero cambio en la acción social, a través de los impactos que se generan dentro y fuera de la organización. En otras palabras, es una nueva forma de entender la responsabilidad que se tiene con las zonas de influencia de la actividad académica (Gómez, 2018).

Vallaeys (2008) considera a la RSU como un concepto de «exploración colectiva», a través del cual se define una nueva forma de gestión en las organizaciones universitarias, entendiendo gestión desde una perspectiva en la cual las personas se organizan, conviven juntas y trabajan en pro de hacer más habitable el mundo. 


\section{Tabla 1 \\ Enfoques de la responsabilidad social universitaria}

\begin{tabular}{|c|c|c|}
\hline \multicolumn{3}{|c|}{ Perspectiva o enfoque: gerencia o directiva } \\
\hline $\begin{array}{l}\text { Analizar el impacto del quehacer } \\
\text { universitario en la sociedad, sobre } \\
\text { todo mediante la rendición de cuentas } \\
\text { de sus acciones y decisiones hacia sus } \\
\text { stakeholders. (Bricall, 200o; Vallaeys et } \\
\text { al., 2009). }\end{array}$ & $\begin{array}{l}\text { Asociación Europea } \\
\text { de Universidades. }\end{array}$ & $\begin{array}{l}\text { Mayor interacción y participación de las partes } \\
\text { interesadas en la gestión de las universidades. } \\
\text { Rendición de cuentas a la sociedad. } \\
\text { Gestión responsable de los impactos de la } \\
\text { universidad. }\end{array}$ \\
\hline \multicolumn{3}{|l|}{ Perspectiva o enfoque: transformacional } \\
\hline \multirow{7}{*}{$\begin{array}{l}\text { Revisar la contribución del quehacer } \\
\text { universitario al necesario debatey } \\
\text { reflexión para alcanzar una sociedad } \\
\text { más sustentable y justa. }\end{array}$} & & $\begin{array}{l}\text { Aprendizaje servicio: formación de ciudadanos } \\
\text { responsables, transformación social. }\end{array}$ \\
\hline & $\begin{array}{l}\text { UNESCO } \\
(1998,2009) \text {. }\end{array}$ & $\begin{array}{l}\text { Responsabilidad de la universidad en la } \\
\text { producción del conocimiento científico } \\
\text { demandado socialmente. }\end{array}$ \\
\hline & Gibbons et al. (1997). & $\begin{array}{l}\text { Enfatiza la incorporación de múltiples actores } \\
\text { al proceso, y una conciencia social de los } \\
\text { problemas a investigar. }\end{array}$ \\
\hline & Kliksberg (2009). & $\begin{array}{l}\text { Liderazgo ético de la universidad, } \\
\text { participación en el debate de temas sociales. }\end{array}$ \\
\hline & Chomsky (2002). & $\begin{array}{l}\text { Rol reflexivo, crítico y propositivo sobre la } \\
\text { sociedad. }\end{array}$ \\
\hline & & $\begin{array}{l}\text { Cooperación universitaria al desarrollo: } \\
\text { transferir capacidades y conocimientos a } \\
\text { países en vías de desarrollo. }\end{array}$ \\
\hline & & $\begin{array}{l}\text { Multiversidad: respuestas académicas a las } \\
\text { crecientes expectativas sociales sobre su } \\
\text { quehacer. }\end{array}$ \\
\hline \multicolumn{3}{|l|}{ Perspectiva o enfoque: normativa } \\
\hline $\begin{array}{l}\text { Desarrollo de marcos valóricos } \\
\text { desde la universidad como un eje } \\
\text { normativo para hacer lo correcto } \\
\text { en la vida en sociedad, mediante } \\
\text { el establecimiento de redes } \\
\text { universitarias nacionales o globales } \\
\text { entorno a la responsabilidad } \\
\text { social. Global Compact PRME. } \\
\text { (Declaración de Talloires, 2005). }\end{array}$ & $\begin{array}{l}\text { Corporación } \\
\text { Participa (2001). }\end{array}$ & $\begin{array}{l}\text { Principios responsables globales: } \\
\text { fortalecimiento de las responsabilidades } \\
\text { sociales y cívicas de la educación superior. }\end{array}$ \\
\hline
\end{tabular}

Fuente: Gaete, R. (2011). La responsabilidad social universitaria como desafío para la gestión estratégica de la educación superior: el caso de España. Revista de Educación, pp. 113-114. 
Vallaeys (2014) sostiene que la RSU «es una nueva política de gestión universitaria para responder a los impactos organizacionales y académicos de la universidad». Por lo tanto, no es «extensión solidaria», sino que debe estar presente en el ser y quehacer de la vida universitaria. Con relación a la importancia de la RSU, Vallaeys (2014) hace referencia a la ISO 26000:2010(es); el propósito de esta norma internacional es «fomentar que las organizaciones vayan más allá del cumplimiento legal, reconociendo que el cumplimiento de la ley es una obligación fundamental para cualquier organización y una parte esencial de su responsabilidad social». (ISO, s.f.).

Para Marí (2014) la RSU es un desafío de las universidades, dado que implica que las instituciones de educación superior se comprometan con una formación integral, donde se formen buenos profesionales y, sobre todo, personas sensibles con el contexto en el que se desarrollan.

Gaete (2011) ofrece un análisis de las diferentes perspectivas y enfoques que analizan el concepto de la RSU con el objetivo de facilitar su «comprensión comodesafío estratégico para las universidades» (véase la tabla 1). Expone tres perspectivas: gerencial o directiva, transformacional y normativa.

En la perspectiva gerencial, el objetivo principal es lograr una mayor interacción y participación de las partes interesadas en la gestión de las universidades. Por lo tanto, se preocupan por el análisis del impacto del quehacer universitario en la sociedad.

En la perspectiva transformacional, se orienta a la revisión del quehacer universitario sobre la base del debate y reflexión para alcanzar una sociedad más sustentabley justa, a través de los procesos de formación, investigación, liderazgo social, compromiso y acción social.

En la perspectiva normativa, la universidad como eje normativo debe desarrollar marcos valóricos para «hacer lo correcto en la vida» en sociedad. Para ello establece redes universitarias nacionales e internacionales en torno a la RSU.

Rozas (2012) señala que es de suma importancia que se definan políticas institucionales que relacionen la responsabilidad social universitaria con el ser y quehacer de la universidad pública.

Por su parte, De la Calle (2010) propone que los futuros profesionales deben ser formados desde una percepción integral, como agentes de cambio frente a los problemas que aquejan en la actualidad a la humanidad, tales como la defensa del ecosistema, así como el respeto por los derechos humanos.

En la Ley $\mathrm{N}^{\circ}$ 30220, Ley Universitaria peruana, en el artículo 124, se enuncia que la RSU es base de la vida universitaria, constructora del desarrollo sostenible, así como del bienestar de la sociedad. Esta definición debe englobar a toda la comunidad universitaria desde las diferentes dimensiones que la componen: académica, investigación, extensión, ambiental, así como institucional (Congreso de la República, 2014).

Además, en la mencionada ley se señala que los procesos de acreditación hacen suyo el enfoque de RSU y lo materializan a través de estándares, en donde se establece que 
las universidades, en sus diferentes escuelas profesionales, deben identificar, definir y desarrollar acciones articuladas con la formación integral de los estudiantes, así como implementar y monitorear políticas ambientales (Sineace, 2017).

Se evidencia la exigencia de demostrar actividades de RSU en el quehacer de la comunidad universitaria. Es por ello que la calidad educativa debe ser un concepto que esté estrechamente ligado a la aplicación de políticas de responsabilidad social universitaria. Como lo menciona Gómez (2018), el concepto de calidad educativa es una noción que va más allá de los muros universitarios, ya que las universidades tienen la responsabilidad de irradiar a las zonas de su influencia el impacto de las actividades que desarrollan.

Podemos afirmar que la RSU debe ser entendida como el compromiso institucional por gestionar el impacto generado en las diversas funciones o actividades que desarrollan en la sociedad las universidades, considerando la importancia de los integrantes de la comunidad universitaria, el cuidado por el medioambiente y el vínculo con otras organizaciones públicas y privadas, con el fin de buscary propiciar el desarrollo sostenible y bienestar de la sociedad.

Baca et al. (2017) proponen un método sistémico para evaluar la RSU. Se diseñan dos instrumentos para analizar la gestión de RSU: una lista de chequeo de 90 ítems y un cuestionario estructurado de 39 ítems. Se muestra que ambos instrumentos son herramientas válidas para evaluar el nivel de cumplimiento de RSU. Los autores concluyen que el $44.4 \%$ de los estándares se cumplieron en una universidad privada de Lima, y que el impacto en las actividades de RSU en los stakeholders internos es de manera significativa en un $37.2 \%$, mientras que el $31.6 \%$ impacta de manera escasa y el $31.19 \%$ no tiene ningún impacto. También determinaron las diferencias significativas en todas las dimensiones (gestión organizacional, gestión ambiental, docencia, extensióne investigación) de la RSU, en los stakeholders internos.

Baca etal. (2017), después deanalizardiferentes propuestas conceptuales, concluyen que la RSU engloba dos conceptos claves: aspectos políticos y capacidades organizacionales.

Castañeda et al. (2007) sostienen que la RSU debe ser definida como una política de calidad institucional sobre el quehacer universitario a través delagestión responsablede los impactos educativos, científico-epistemológicos, organizacionales y sociales quegenera la universidad.

Vallaeys (2008) y Bacigalupo (2008, citado en Baca et al., 2017) afirman que la RSU es una capacidad que tiene la universidad de difundir y poner en práctica su razón de ser (misión), por medio de cuatro procesos claves: gestión, docencia, investigación y extensión.

La definición de RSU de Baca et al. (2017) se recoge en la figura 1. Puede observarse que la RSU engloba los aspectos esenciales dentro de una institución de formación académica (docencia, extensión, gestión, investigación y medioambiente). Estas áreas tienen contenidos transversales que dirigen su acción y que provienen de los valores, políticas y estrategias de RSU de la alta dirección, propuestos en la fase de planificación. El fin último de 


\section{Figura 1}

\section{Definición integral de responsabilidad social universitaria}
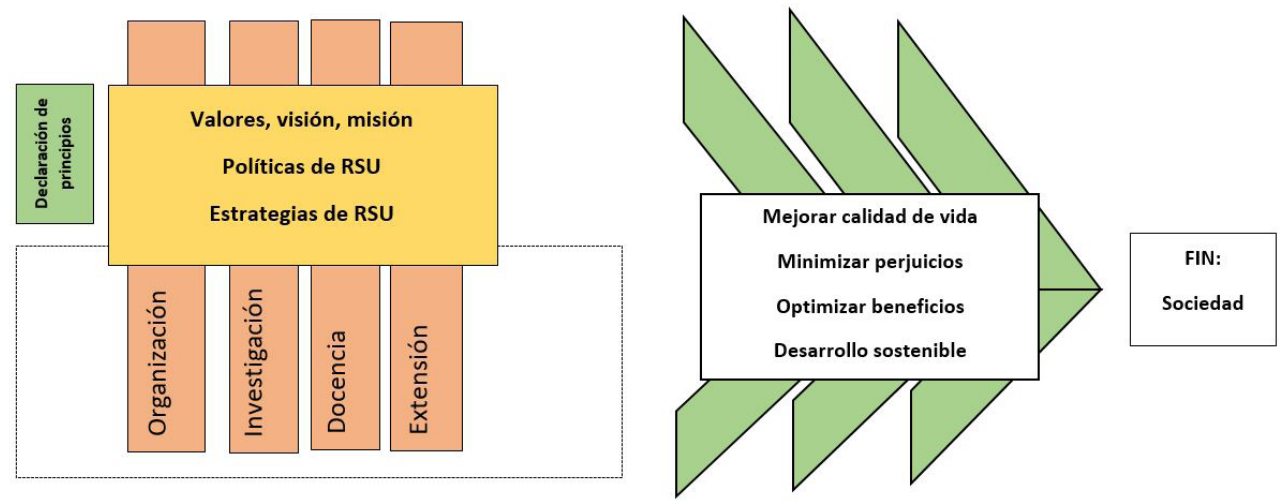

Fuente: Baca, Rondán y García (2017, p. 12).

lo expresado es mejorar la calidad de vida de la población, minimizar los impactos perjudiciales de la universidad y optimizar los beneficios.

En la tabla 2 se presenta un resumen con las definiciones conceptuales de las dimensiones de RSU definidas por Baca et al. (2017).

Dentro de esta visión global de RSU, para Baca et al. (2017) la dimensión que comprende lo relacionado al medio ambiente es transversal a todas las descritas anteriormente y es entendida dentro del concepto de «universidad sostenible». Esta comprende: gestión medioambiental y marketing medioambiental responsable.

Por lo acotado anteriormente, se formula la siguiente pregunta de investigación: ¿cuál es el nivel de cumplimiento de los estándares de RSU —según el método sistémico propuesto por Baca etal. (2017) - ylainteracción conactividades de RSU por parte de los stakeholders internos (docentes, alumnosy colaboradores) de la Escuela Profesional de Administración de una universidad privada de Arequipa?

\section{Aspectos metodológicos}

El método sistémico de Baca et al. (2017) tiene un enfoque de tipo mixto: cualitativo porque se entrevistó a las autoridades de la Escuela de Administración (Director de Escuela de Administración, Director de Departamento y Decano de la Facultad) y cuantitativo porque se presentan datos estadísticos. La información estadística se obtuvo a partir de la aplicación de dos instrumentos: uno dirigido a autoridades de la escuela, utilizando una lista de chequeo estructurado con 90 ítems a medir, los cuales tienen tres opciones de respuesta tipo semáforo: no cumple (rojo), cumple parcialmente (amarillo) y cumple con el estándar (verde); y el otro instrumento fue un cuestionario estructurado autoaplicativo en escala de Likert de cuatro niveles (nunca/no sé, pocas veces, muchas 


\section{Tabla 2 \\ Dimensiones de la responsabilidad social universitaria}

\begin{tabular}{|c|c|}
\hline Dimensiones & Concepto y características \\
\hline $\begin{array}{l}\text { Gestión } \\
\text { organizacional } \\
\text { y ambiental }\end{array}$ & $\begin{array}{l}\text { Comprende el impacto organizacional, se divide en impactos laborales y ambientales. Debido } \\
\text { a que la universidad emplea personas contratadas o nombradas, genera un impacto laboral. La } \\
\text { meta se orienta hacia la transformación de la universidad en una pequeña comunidad ejemplar } \\
\text { de democracia donde valores como la transparencia sean fomentados y difundidos. En esta } \\
\text { dimensión, herramientas como las ISO son útiles para alcanzar estándares de calidad. La idea es } \\
\text { fomentar en la universidad un espacio de aprendizaje donde se desarrolle un tipo de enseñanza } \\
\text { doble: en la universidad los estudiantes aprenden su carrera profesional y aprenden también } \\
\text { los hábitos y valores ciudadanos; adquiriéndose esto sobre todo en la práctica cotidiana de } \\
\text { principios y hábitos comunes. El personal que trabaja en la institución educativa (asistentes, } \\
\text { administrativos, directores, docentes, rectores) contribuye en el establecimiento de estándares } \\
\text { operacionales relacionados al contenido curricular de estudios, entre otras habilidades que } \\
\text { permiten el desarrollo de estudiantes competentes en el mercado laboral. El éxito de una } \\
\text { universidad que es socialmente responsable dependerá también de la gestión de personas, la } \\
\text { resolución de conflictos y el aseguramiento del comportamiento ético. }\end{array}$ \\
\hline
\end{tabular}

Docencia La dimensión de docencia socialmente responsable hace referencia a los impactos educativos que presenta la universidad en alumnos y docentes. Esta dimensión debe enfocarse en la capacitación de docentes bajo el enfoque de la responsabilidad social universitaria, promoviendo el desarrollo de materias y proyectos de carácter social, abriendo las clases a un espacio de enseñanza práctica donde se fomente la resolución de problemas sociales mediante propuestas innovadoras. La idea es impulsar la creatividad, tanto en cursos como en proyectos y prácticas profesionales. El estudiante debe aprender a realizar cosas útiles que a su vez le permitan formarse como ciudadano y parte de una comunidad. Esto promoverá la instauración de una mayor articulación entre disciplinas de aprendizaje (enfoque multidisciplinario e interdisciplinario) para tratar los problemas sociales y una mayor articulación entre la docencia, la investigación y la proyección social.

Extensión Se enfoca en trabajar fomentando la fusión de la investigación y el trabajo con docentes, para implementar y administrar proyectos y programas de desarrollo que puedan ser fuente de investigación aplicada, tanto para la universidad como para la comunidad. Se debe promover una vinculación clave con los actores sociales para el desarrollo. Estas iniciativas deben estar sistematizadas dentro de la organización institucional y académica de la universidad. Por ello, Vallaeys (2004) sostiene que es importante lograr la integración de la proyección social en el corazón de la institución educativa, a través de una instancia específica dentro de la universidad que se encargue de gestionar las iniciativas estudiantiles y docentes, sin dejar de lado el control de la calidad. Tal unión entre gestión organizacional, proyección social, docencia e investigación promoverá el aumento significativo del voluntariado estudiantil, lo que se proyectará en la formación académica y humana.

Investigación Esta dimensión está orientada a promover la investigación para el desarrollo en todas las materias y ciencias que la universidad fomente con su estudio. La investigación que se realice en la universidad debe guardar relación con los problemas sociales y ser, a su vez, interdisciplinaria. Los conocimientos generados deben tener relevancia social y ser accesibles a la comunidad, esto debe desplegarse siempre teniendo en consideración el respeto de los derechos humanos y consideraciones éticas. Dentro de las estrategias para el fomento de esta dimensión encontramos la firma de convenios con actores estratégicos, como municipalidades, organizaciones gubernamentales y no gubernamentales (ONG), donde se invite a los departamentos de las carreras a promover investigaciones interdisciplinarias aplicadas a dichas localidades. Así, investigadores y docentes se encontrarían en el mismo lugar, trabajando sobre la misma problemática, desde sus especialidades respectivas, creando una sinergia de conocimientos e interdisciplinariedad.

Fuente: Baca (2015). 
veces y siempre) con 39 ítems a medir, el cual se aplicó a docentes, alumnos y colaboradores de la Escuela de Administración, con una fiabilidad del Alfa de Cronbach de o.954.

La investigación es de nivel descriptivo, ya que busca «describir fenómenos, situaciones, contextos y sucesos: esto es, detallar cómo son y cómo se manifiestan» (Hernández et al., 2014, p. 92).

Para el análisis de la lista de chequeo estructurado, se presentan los porcentajes alcanzados por cada dimensión. Para el cuestionario autoaplicativo, se utilizó el software estadístico SPSS 25.

\section{Resultados}

En las figuras 2 y 3 se muestran los resultados de la aplicación de la lista de chequeo a las autoridades de la Escuela de Administración de la UPA.
En la figura 2 se evidencia un avance poco significativo en la RSU: el 42.86\% (rojo) de los estándares no son cumplidos, el $41.76 \%$ (amarillo) de los estándares son considerados como oportunidades de mejora y el $15.38 \%$ (verde) se cumplen plenamente.

Con relación al no cumplimiento de los estándares de la RSU (rojo), los resultados de Baca et al. (2017) en una universidad privada de Lima fueron diferentes a los obtenidos en la presente investigación. Sin embargo, respecto a las oportunidades de mejora de la RSU (amarillo), se coincide con los resultados de Baca et al. (2017). Con relación al cumplimiento de los estándares de la RSU (verde) de las autoridades, se difiere de los resultados de Baca et al. (2017), los cuales muestran un avance en los estándares de RSU en comparación con la UPA.

\section{Figura 2}

\section{Cumplimiento de indicadores de RSU de acuerdo con las autoridades (Porcentaje de indicadores según nivel de cumplimiento)}

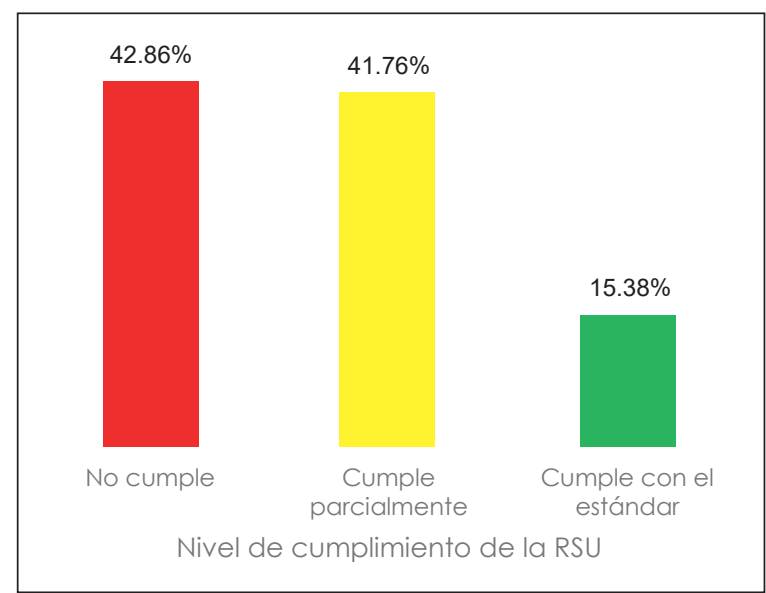

Fuente: Encuesta de las autoras a autoridades de la Escuela de Administración de la UPA basada en Lista de Chequeo UPA-RSU. 
Figura 3

\section{Cumplimiento de indicadores de RSU, según dimensión (\%)}

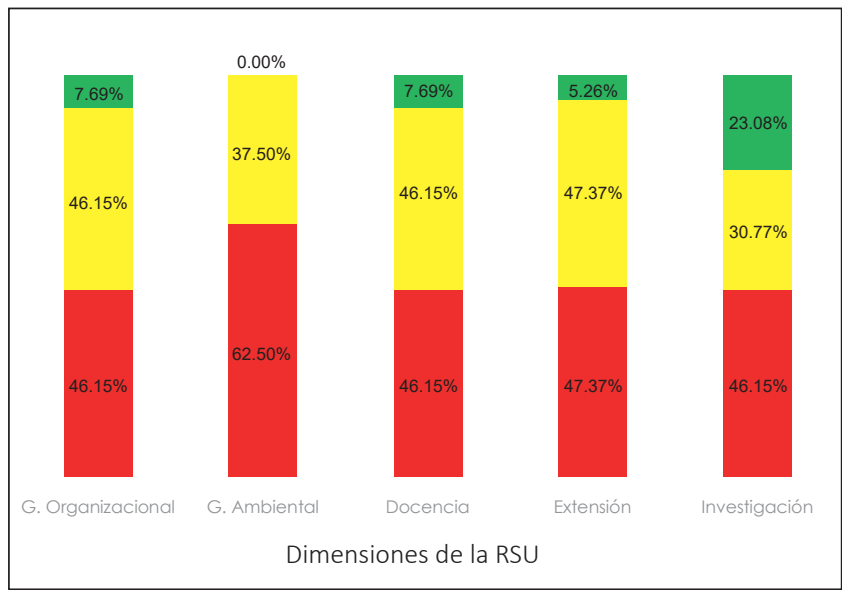

Fuente: Encuesta de las autoras a autoridades de la Escuela de Administración de la UPA basada en Lista de Chequeo UPA-RSU.

La figura 3 muestra los resultados de cumplimiento según dimensión de la RSU. Las dimensiones gestión organizacional y docencia tuvieron el mismo comportamiento, el 7.69\% cumple con los estándares (verde), el $46.15 \%$ tiene oportunidades de mejora (amarillo) y el $46.15 \%$ no cumple con los estándares (rojo). Los resultados difieren de los de Baca et al. (2017), en donde el cumplimiento de los estándares (verde) es en gestión organizacional $(59.5 \%)$ y en docencia ( $46.15 \%)$. La dimensión gestión ambiental es preocupante, ya que los estándares no cumplidos representan un $62.50 \%$ (rojo), solamente el $37.50 \%$ de los estándares muestran oportunidades de mejora (amarillo) y el cumplimiento con el estándar (verde) es de o\%. Los resultados difieren de los de Baca et al. (2017), que obtuvo un $37.50 \%$ de cumplimiento (verde) y un $25 \%$ en oportunidades de mejora (amarillo). En extensión, el 5.26\% cumple con los estándares (verde), un
$47.37 \%$ tiene oportunidades de mejora (amarillo) y el $47.37 \%$ no cumple con los estándares (rojo).

Estos resultados difieren de los de Baca et al. (2017), donde el cumplimiento de los estándares fue de $31.6 \%$ (verde), superior al de la presente investigación (5.26\%). La dimensión investigación tiene un mejor resultado en el cumplimiento de los estándares, con un $23.08 \%$ (verde), el $30.77 \%$ tiene oportunidades de mejora (amarillo) y el $46.15 \%$ no cumple (rojo).

En la tabla 3 se presentan los resultados del cuestionario sobre la interacción de los stakeholders internos de la UPA (docentes, alumnos y colaboradores) con actividades de RSU, según dimensión. Los tamaños de muestra recogidos son de 110 docentes, 304 alumnos y 36 colaboradores, con un total de 450 encuestados. Para los resultados se obtuvo un Alfa de Cronbach de 0.951. 


\section{Tabla 3}

Interacción de los stakeholders internos con actividades de RSU, según dimensión

\begin{tabular}{lcccc}
\hline \multicolumn{1}{c}{ Dimensiones de RSU } & \multicolumn{3}{c}{ Frecuencia de interacción (\%) } \\
& Nunca & Poco & Mucho & Siempre \\
\hline Gestión organizacional & 10.76 & 19.92 & 43.86 & 25.47 \\
Gestión ambiental & 28.50 & 27.07 & 32.49 & 11.94 \\
Docencia & 10.15 & 26.65 & 42.93 & 20.27 \\
Extensión & 21.33 & 30.42 & 35.98 & 12.27 \\
Investigación & 16.87 & 30.17 & 40.58 & 12.38 \\
RSU de la universidad & 17.52 & 26.85 & 39.17 & 16.46 \\
\hline
\end{tabular}

Fuente: Elaboración propia basada cuestionario UPA-RSU.

La interacción con actividades de RSU, en conjunto, es la siguiente: el $55.63 \%$ interactúa mucho o siempre, el $26.85 \%$ interactúa poco y el $17.52 \%$ no interactúa nunca, diferenciándose de los resultados obtenidos por Baca et al. (2017) que fueron de un $37.2 \%$ (mucho y siempre), $31.6 \%$ (poco) y $31.19 \%$ (nunca).

La interacción con actividades de RSU es mayor para la dimensión gestión organizacional, con un $69.33 \%$ de encuestados reportando que interactúan mucho o siempre con actividades en esta dimensión. Le siguen las dimensiones docencia, con un 63.20\%; investigación, con 52.96\%; extensión, con $48.25 \%$, y gestión ambiental, con $44.43 \%$.

La proporción de encuestados que señalan que nunca interactuaron con actividades de RSU es mayor para la dimensión gestión ambiental, con un $28.50 \%$. Le siguen las dimensiones extensión, con un $21.33 \%$; investigación, con un $16.87 \%$; gestión organizacional, con un $10.76 \%$, y docencia, con un $10.15 \%$.

En la tabla 4 se presenta la comparación de medias en los stakeholders internos por dimensión, al $95 \%$ de nivel de confianza.

Las dimensiones que obtuvieron un valor $\mathrm{p}$ ( $p$-value) menor a 0.05 son gestión organizacional (o.0oo), gestión ambiental (o.oo1) y docencia (o.040), lo que implica la existencia de diferencias significativas entre las medias de interacción de los stakeholders internos en las dimensiones citadas. Por otro lado, las dimensiones que obtuvieron un valor $\mathrm{p}$ mayor a 0.05 fueron extensión (o.109) e investigación (o.085), lo que implica la no existencia de diferencias significativas entre las medias de interacción de los stakeholders internos en las dimensiones citadas. Estos resultados contrastan con los obtenidos por Baca et al. (2017), con valores p menores a 0.05 en las dimensiones extensión (o.0oo) e investigación (o.ooo). 
Tabla 4

Nivel de interacción con actividades de RSU, según dimensión de RSU y categoría del encuestado

\begin{tabular}{|c|c|c|c|c|c|}
\hline & & $\mathrm{N}$ & Media & $\mathrm{F}$ & $p$-value \\
\hline \multirow{4}{*}{ Gestión organizacional } & Docente & 110 & 28.88 & \multirow{4}{*}{11.177} & \multirow{4}{*}{0.000} \\
\hline & Alumno & 304 & 27.14 & & \\
\hline & Colaborador & 36 & 31.92 & & \\
\hline & Total & 450 & 27.95 & & \\
\hline \multirow{4}{*}{ Gestión ambiental } & Docente & 110 & 7.45 & \multirow{4}{*}{6.721} & \multirow{4}{*}{0.001} \\
\hline & Alumno & 304 & 8.71 & & \\
\hline & Colaborador & 36 & 9.17 & & \\
\hline & Total & 450 & 8.44 & & \\
\hline \multirow{4}{*}{ Docencia } & Docente & 110 & 19.11 & \multirow{4}{*}{3.253} & \multirow{4}{*}{0.040} \\
\hline & Alumno & 304 & 18.93 & & \\
\hline & Colaborador & 36 & 20.83 & & \\
\hline & Total & 450 & 19.13 & & \\
\hline \multirow{4}{*}{ Extensión } & Docente & 110 & 19.75 & \multirow{4}{*}{2.225} & \multirow{4}{*}{0.109} \\
\hline & Alumno & 304 & 18.81 & & \\
\hline & Colaborador & 36 & 20.00 & & \\
\hline & Total & 450 & 19.14 & & \\
\hline \multirow{4}{*}{ Investigación } & Docente & 110 & 23.71 & \multirow{4}{*}{2.480} & \multirow{4}{*}{0.085} \\
\hline & Alumno & 304 & 25.18 & & \\
\hline & Colaborador & 36 & 25.53 & & \\
\hline & Total & 450 & 24.85 & & \\
\hline
\end{tabular}

Fuente: Elaboración propia basada cuestionario UPA-RSU.

\section{Conclusiones}

El método sistémico de Baca utiliza, en primer lugar, una aproximación cualitativa para obtener información detallada sobre las diversas labores y cometidos de RSU; luego, utiliza una aproximación cuantitativa para obtener data estadística sobre el nivel de conocimiento que tienen los stakeholders internos (docente, alumno y colaborador) de las diversas actividades de RSU.
En el caso de una universidad privada de Arequipa, las autoridades de la Escuela de Administración perciben que el $42.86 \%$ (rojo) de los estándares no se cumplen, el 41.76\% (amarillo) de los estándares son considerados como oportunidades de mejora y el $15.38 \%$ (verde) se cumplen plenamente.

La interacción de las actividades de RSU con los stakeholders internos en la Escuela Profesional de Administración se da 
mucho o siempre según el $55.63 \%$ de los encuestados, el $26.85 \%$ tiene poca interacción y el $17.52 \%$ no interactúa nunca.

Las dimensiones para las que se observan diferencias significativas en las medias entre las distintas categorías de stakeholders internos son gestión organizacional, gestión ambiental y docencia.

La revisión de las dimensiones propuesta por Baca en su modelo sistémico en una universidad privada de Arequipa sugieren que las universidades tienen aún un camino que recorrer en la definición de políticas de responsabilidad social universitaria y en el alineamiento estratégico de las actividades de las distintas dimensiones de RSU (gestión organizacional, gestión ambiental, docencia, extensión e investigación).

\section{Referencias}

Baca, H. (2015). La responsabilidad social universitaria: propuesta conceptual y medición en el ámbito de una universidad privada de Lima-Perú. (Tesis doctoral). Sevilla: Universidad de Sevilla.

Baca, H., Rondán, F., y García, J. (2017). Propuesta de medición de la responsabilidad social universitaria. Espacios, 38(43).

Castañeda, G., Ruiz, M., Viloria, O., Castañeda, R., y Quevedo, Y. (2007). El rol de las Universidades en el Contexto de la Responsabilidad Social Empresarial. Revista NEGOTIUM Ciencias Gerenciales, 3(8), 105.

Congreso de la República. (3 de julio de 2014). Ley Universitaria Nro. 30220.
De la Calle Maldonado, C. (2010). La formación de la responsabilidad social del universitario: un estudio empírico. (Tesis doctoral). Madrid: Universidad Complutense de Madrid.

Domínguez Granda, J., y Rama, C. (2012). La Responsabilidad Social Universitaria en la Educación a Distancia. Chimbote: ULADECH Católica.

Gaete, R. (2011). La responsabilidad social universitaria como desafío para la gestión estratégica de la Educación Superior: el caso de España. Revista de Educación, 109-133.

Gaete, R. (s.f.). Responsabilidad Social Universitaria: una nueva mirada a la relación de la Universidad con la sociedad desde la perspectiva de las partes interesadas. Un estudio de caso. (Tesis doctoral). Valladolid: Universidad de Valladolid.

Gil, F. (2013). La responsabilidad social universitaria desde la perspectiva ambiental: universidad y desarrollo sustentable. (Tesis de maestría). México: Universidad Nacional Autónoma de México.

Gómez, S. (2018). La calidad educativa en el ámbito universitario. Arequipa: Sandra J. Gómez Rodríguez de Salinas.

Hernández, R., Fernández, C., y Baptista, P. (2014). Metodología de la Investigación (sexta ed.). México: Mc Graw Hill.

ISO. (s.f.). ISO 2600o:2010(es) Guía de responsabilidad social. Recuperado el 23 de enero de 2019, de Online Browsing Platform Website: https://www.iso.org/obp/ ui\#iso:std:iso:26ooo:ed-1:v1:es 
Juan Pablo II. (1990). Ex Corde Ecclesiae. Vaticano: Don Bosco.

Marí, E. (2014). Responsabilidad Social Universitaria en la Universitat Politècnica de València: Propuestas para su desarrollo. (Diplomatura). Valencia: Universitat Politècnica de València.

Mendoza, D., y Salas, E. (2015). Responsabilidad Social Universitaria, una oportunidad para generar la calidad académica en la Universidad de la Guajira. Colombia. Memorias, Congreso Global de Investigadores, 1, 83-98.

Rozas, A. (2012). La responsabilidad social como herramienta de la auditoría en las universidades públicas de Lima. (Tesis de maestría). Lima: Universidad Nacional Mayor de San Marcos.
SINEACE. (2017). Modelo de acreditación para programas de estudios de educación superior universitaria. Lima: Tarea asociación gráfica educativa.

UNESCO. (2009). Cátedras UNESCO. Recuperado el 15 de mayo de 2019, de UNESCO Website: http:// www.unesco.org/new/es/mexico/ communities/unesco-chairs/

Vallaeys, F. (2008). Responsabilidad Social Universitaria: una nueva filosofía de gestión ética e inteligente para las universidades. Revista de Educación Superior y Sociedad, 13(2), 195-219.

Vallaeys, F. (2014). La responsabilidad social universitaria: un nuevo modelo universitario contra la mercantilización. Universia, 5(12), 105-117.

Fecha de recepción: 8 de julio de 2019 Fecha de aceptación: 5 de noviembre de 2019 